by GPs and hospitals. $<50 \%$ took action when concordance reports showed variance (under or over use) from prescribed treatment.

2. Lack of ability to detect hypoxic patients with $>20 \%$ community healthcare sites not having access to an oximeter.

3. Fire safety officers are rarely advised about the persistent smoker (only 16\%) despite the potential risk to patients, their families and the general public. Local guidance on appropriate steps to take is rare (35\%). 3 HOS units denied LTOT for smokers and one assessed this by exhaled carbon monoxide measurement.

4. A variety of methods for protecting patients from excessive oxygen are favoured but use appears limited. When asked what policy respondents favoured, universal precaution (as promoted by ambulance guidelines) was most popular (60\%) while $20 \%$ favoured oxygen cards and $20 \%$ patient specific protocols (PSP).

5. A specific local policy for removing oxygen when no longer indicated or used is rare $(<25 \%)$. This, coupled with inadequate follow-up of patients started on oxygen during hospital admission, suggests significant waste with the current oxygen provision.

6. Respondents indicated guidance on oxygen removal, contract monitoring, assessment for ambulatory oxygen and training in arterial or capillary blood gases as being required.

Conclusions Problems in healthcare coordination, public and patient safety and in removing oxygen once ordered were common. There is a need to integrate hospital and community teams and to prepare for safe mobilisation and contract management so that a quality home oxygen service can be provided in the future.

\section{P103 THE PRACTICE OF PRESCRIPTION OF LONG-TERM OXYGEN THERAPY TO PATIENTS WHO CONTINUE TO SMOKE}

doi:10.1136/thoraxjnl-2011-201054c.103

C K Y Lee, J Noble, D A Wales, R M Jones. Nevill Hall Hospital, Abergavenny, UK

Introduction Although patients who receive long-term oxygen therapy (LTOT) are discouraged from smoking, a substantial proportion of active smokers receive this therapy. There is limited published guidance addressing this issue.

Methods The authors designed a questionnaire consisting of six multiple choice type questions based on the practice of the prescription of LTOT, particularly focusing on the provision of LTOT to active smokers. This was sent to all consultant respiratory physicians in Wales.

Results 35 out of 45 (73\%) questionnaires were returned. 17 physicians (49\%) did not prescribe LTOT to active smokers, whereas $18(51 \%)$ did. For those who did not prescribe LTOT to smokers, this was according to departmental policy in 9 (53\%) and personal practice in $8(47 \%)$. In this group, all respondents stated the reason for not prescribing oxygen was due to risk of harm to the individual, with the majority (88\%) also citing risk to other household members. Other reasons included the reduced likelihood of benefit and effectiveness in smokers (35\%) and as an incentive to smoking cessation. 7 (41\%) of this group would consider prescribing oxygen to smokers as palliation for severe hypoxia and malignancy. Of the physicians who prescribed LTOT to active smokers, 16 (89\%) felt there was existing evidence suggesting benefit in both smokers and non-smokers. $10(55 \%)$ of this group considered it unethical to deny patients LTOT and 11 (61\%) felt it was the responsibility of individual patients. LTOT was prescribed only after risk and capacity assessment. Contraindications to prescribing LTOT in this group included cognitive impairment and inability to comprehend the risks. $22(63 \%)$ regularly monitored patients' smoking status while on LTOT. Of these, the majority (68\%) did so by history alone. 6 (27\%) performed Carbon Monoxide monitoring. 31 respondents $(89 \%)$ routinely counselled patients on risks of fire and burns with continued smoking on LTOT. 22 respondents (63\%) had seen burns or injuries from smoking with LTOT on at least one occasion.

Conclusion There are substantial variations in practice among respiratory physicians across Wales and likely nationwide. National guidance on this particular issue needs to be addressed to ensure standardisation of care.

\section{P104 SOUTHAMPTON CITY: FINDING THE MISSING MILLIONS AND REDUCING ADMISSIONS THROUGH MEDICAL INTELLIGENCE}

doi:10.1136/thoraxjnl-2011-201054c.104

S C Bourne, T Wikinson, H Kruk, K Austin, J Watson, N James. Southampton University Hospitals NHS Trust, Southampton, UK

Background Southampton City PCT was highlighted in a BLF report as the only hotspot on the South Coast with a population at high risk of hospital admissions with an acute exacerbation of COPD (AECOPD). The reasons stated were a low number of registered COPD patients $(1.5 \%)$, high smoking prevalence $(32 \%)$ and deprivation. Predicted levels of COPD from local healthcare modelling were $6 \%$. The trust was also experiencing a year on year increase of $15 \%$ in admissions for an acute exacerbation of COPD.

Method Southampton University Hospitals developed a primary care support team led by consultant respiratory physicians with support from respiratory nurse specialists to support primary care in the diagnosis and management of COPD. The education was delivered in a variety of formats from interactive lectures to groups of primary care doctors and nurses, small spirometry educational sessions with practice nurses, within practice education, locally led COPD diploma modules, and the development of a website (http:// www.copdeducation.org.uk). The audit was performed on all admissions coded as COPD Exacerbation from the last 3 years and all notes reviewed to confirm diagnosis.

Results During the year long project COPD prevalence (from QOF) rose from $1.5 \%$ to $2.27 \%$; an increase of $50 \%$. The audit revealed several trends (1) admissions through the ED department rose by 10\% each year from 2007 to 2010; (2) patients increasingly self presented to secondary care and (3) a large proportion of the admissions $(22 \%)$ were due to a small number of frequent attendees (34 patients responsible for 176 admissions). We cohorted the frequent attendees and with a combination of discovery interviews and nursing/clinical support reduced admissions in this cohort by $78 \%$ ). During this period COPD admissions reduced by $19 \%$ and 30 day readmission reduced from $13 \%$ to just $1.7 \%$ !

Conclusion Through the process of primary care education supported with other teaching modalities the diagnosis of COPD improved in Southampton. Medical intelligence of the admitted population combined with an appropriate intervention can reduce COPD admissions. This is just one of the ways the finding the missing millions part of the national strategy can be delivered.

Funding The Health Foundation (SHINE).

\section{P105 DIRECT ACCESS PULMONARY FUNCTION TESTING FOR PRIMARY CARE}

doi:10.1136/thoraxjnl-2011-201054c.105

${ }^{1} \mathrm{~K}$ E Backler, ${ }^{1} \mathrm{~L}$ Leech, ${ }^{1} \mathrm{M}$ Purdon, ${ }^{2} \mathrm{D}$ Roberts, ${ }^{3} \mathrm{Z}$ Lord, ${ }^{1} \mathrm{R}$ C Buttery. ${ }^{1}$ Hinchingbrooke Hospital NHS Trust, Huntingdon, UK; ${ }^{2}$ Great Staughton Surgery, Great Staughton, UK; ${ }^{3}$ National Lung Improvement Programme, London, UK

Hinchingbrooke Hospital is a Diagnostic pilot site for the NHS Lung Improvement Programme, providing direct access from local GP 